\title{
Research on Body Type Correction of FINA Diving Difficulty Coefficient Based on Rigid Body System
}

\author{
Su Zhang $\left(\mathbb{D},{ }^{1}\right.$ Wen Xiang, ${ }^{2}$ and Guozhong Zou ${ }^{3}{ }^{3}$ \\ ${ }^{1}$ Department of Physical Education, Anhui University of Finance and Economics, Bengbu 233030, China \\ ${ }^{2}$ School of Statistics and Applied Mathematics, Anhui University of Finance and Economics, Bengbu 233030, China \\ ${ }^{3}$ School of Physical Education and Humanities, Nanjing Sport Institute, Nanjing 210014, China \\ Correspondence should be addressed to Guozhong Zou; mynzgz5416@sina.com
}

Received 1 February 2021; Revised 28 February 2021; Accepted 26 March 2021; Published 5 April 2021

Academic Editor: M. Irfan Uddin

Copyright (C) $2021 \mathrm{Su}$ Zhang et al. This is an open access article distributed under the Creative Commons Attribution License, which permits unrestricted use, distribution, and reproduction in any medium, provided the original work is properly cited.

In order to reduce the influence of athlete's body shape on the difficulty coefficient of diving, a more reasonable calculation method of body shape correction coefficient is proposed based on the original calculation rules of diving difficulty coefficient. First, the composition of the original diving difficulty coefficient and influencing factors is analyzed and the relationship between the various structural parts is fully clarified. Second, a coupled nonrigid body dynamics model is established and a 2-body model is used to simulate complex diving actions, and it is concluded that diving time is positively correlated with body shape. Finally, the air movement part and the water entry part of diving are discussed separately, the calculation model of the difficulty coefficient of body shape correction is established, and the original difficulty coefficient is corrected. The results show that the difficulty coefficient of each movement is obviously increased. This effectively avoids the influence of body shape on the diving difficulty coefficient.

\section{Introduction}

Diving is a beautiful water sport. It is a sport in which the athlete jumps into the water in various postures from high places or jumps from diving equipment, completes certain postures in the air, and enters the water with specific movements. In diving competitions, athletes will perform aerial posture changes in the air, such as bending, straight body, knee tuck, and other postures. As the aerial posture changes, the overall moment of inertia changes, and the speed and angle of tossing and turning are also accompanied in order to achieve the purpose of controlling the posture of the bent body in the air [1].

Usually, when judging diving, mainly take two factors into consideration, rotation and aesthetics. The two basic forms of air rotation for athletes are tossing and turning [2]. Tossing is a rotation about a horizontal axis perpendicular to the direction of travel, while a swivel is a rotation about the longitudinal (head to toe) of the athlete's body [3]. The main factors affecting the athlete's completion of the aerial rotation are initial posture, potential energy during take-off, angular velocity, adjustment of the aerial posture, and the characteristics of the athlete's own mechanics and physiological functions. Many factors work together to form a very complex interactive relationship [4].

The movement difficulty coefficient of technical sports reflects the difficulty of its movement. The degree of difficulty of the action is reflected in the angle (or number of turns), the athlete completes in the air to toss and turn. The correct completion of the aerial action and the angle (or numbers of laps) of the completed throw and turn, as well as the perfect landing (water, net) after completing the aerial action, are important basis for evaluating athletes to obtain high scores.

Diving athletes' competition performance depends on the referee's score. The International Swimming Federation (FINA) gives the corresponding difficulty coefficients for different diving actions. The referees in the competition will calculate according to a certain formula based on the performance of the athletes' performance in completing the 
moves and the effect of entering the water. The athlete's completion score for the action, which is multiplied by the difficulty coefficient of the action, is the final score of the action for the athlete. It is generally believed that "the ideal performance has the best size and proportion" [5]. When analyzing somersaults from the perspective of dynamics, "size and proportion" is converted into the inertia of each body part. Due to ethnic differences of contestants from different countries, the athlete's body type (height and weight) will be affected by innate factors, and the same diving action has different requirements for different body types of players. Studies have shown that lightweight athletes have a body type advantage when completing diving [6]. If the difficulty of completing the tossing turn is related to body type, then the difficulty coefficient of the diving movement prescribed by the FINA may not reflect fairness, and it is necessary to correct the existing difficulty coefficient.

This article attempts to combine biological theory and physics knowledge to establish a coupled nonrigid body dynamics model to explore the relationship between diving time and body shape. Respectively, study the aerial part and the water part of diving to establish a body shape correction difficulty coefficient calculation model, revise the original difficulty coefficient, aiming to better ensure the fairness of the competition, and at the same time, provide athletes and coaches with references when they engage in the training of diving of high degree of difficulty.

\section{Basic Assumptions}

In order to solve the problem, we make the following assumptions for certain conditions:

(1) It is assumed that every athlete can complete diving with various difficulty coefficients

(2) It is assumed that the compilation of the difficulty coefficient table is directly related to the standard height and weight of divers

(3) It is assumed that the initial angular momentum obtained by each athlete is the same

(4) Assuming that the ratio of the athlete's upper and lower body is set to $1: 1$, the length of the bend roll is $h / 2$ and the length of the tuck roll is $h / 2$

\section{Noun Explanation and Symbol Explanation}

\subsection{Noun Explanation}

3.1.1. Angular Velocity. In a circle with radians as the unit, the radians traveling in a unit time is the angular velocity and the unit is radians/second.

3.1.2. Angular Momentum. In physics, it is a physical quantity related to the displacement and momentum of an object to the origin. It represents the speed at which the particle vector traverses the area or the violent degree of rigid body rotation on a fixed axis.
3.1.3. Rotor. Rotor refers to a rotating body supported by bearings. For example, an object without a rotating shaft, such as an optical disc, can be regarded as a rotor when it adopts a rigid connection or an additional shaft.

3.1.4. The Moment of Inertia. The moment of inertia is a measure of inertia of a rigid body when it rotates around an axis. It only depends on the shape, mass distribution, and position of the axis of the rigid body and has nothing to do with the state of rotation of the rigid body around the axis (such as the magnitude of angular velocity).

3.2. Symbol Description. The main variables involved in this article are (1) I represents the moment of inertia under the central body frame; (2) $J$ represents the moment of inertia under the main shaft frame; (3) $T^{s}$ represents the pure somersault action time; (4) $T^{t}$ represents the time used for the turning action; (5) $S_{k}$ represents the k-th stage after the decomposition of the diving action; (6) $\tau_{k}$ represents the time used for the k-th stage; (7) $I^{s}$ is the moment of inertia of the somersault action; (8) $I^{t}$ represents the moment of inertia of the turning action; (9) $T$ represents the total time from the start of the air movement to the water entry, that is, the free fall time; (10) $T_{\text {air }}$ represents the time for the athlete to complete the air movement, which is about the function of $h$ and $m$; (11) $T_{\text {water }}$ represents the time from the completion of the movement to the time the athlete enters the water; (12) $h$ represents the athlete's height; (13) $M$ represents the athlete's weight; (14) $T_{\text {air }}^{0}$ indicates the time it takes for athletes of standard height and weight to complete the air movement; and (15) $T_{\text {water }}^{0}$ represents the time from the completion of the movement to the water entry of the athlete under the standard height and weight.

\section{Analysis of the Composition and Influencing Factors of Diving Difficulty Coefficient}

4.1. Analysis of the Problem. FINA has determined the corresponding action code and difficulty coefficient for each diving action in the diving competition rules, which are determined to accord to the differences in action group, competition item, equipment height, action posture, and the number of flip turns. The degree of difficulty is related to the diving athlete's take-off method (the athlete's frontal face and the direction of the somersault during the take-off) and the aerial movements (the number of somersault and twist and the body posture). When scoring, the referees give an action score from 10 to 0 according to the athlete's performance in completing the action and the effect of the water entry. Then, the athlete's completion score is calculated for the action according to a certain formula, and this completion score is multiplied by the action's score; the difficulty factor is the final score of the action of the athlete.

In order to deeply analyze the determination rules of the FINA 10-meter platform diving difficulty coefficient, this research firstly sorts out the action elements corresponding to the diving difficulty coefficient, clarifies the relationship 
between each element, and then analyzes the difficulty coefficient of each part on this basis, and the rules of the difficulty coefficient of the specific movements of the 10meter platform are explained.

\subsection{Solve the Problem}

4.2.1. The Composition of Diving Difficulty Coefficient. The difficulty coefficient of diving is essentially a measure of the difficulty of diving. It firstly divides the difficulty of the action into five parts in a qualitative way from a logical point of view, then regularly gives a number of necessary quantitative standards for the subitems and subelements of each part, applies scientific calculation methods to give each the magnitude of a subitem, and finally adds up to find the degree of difficulty. These five parts are somersault, air posture, twist, take-off method, and unnatural entry into the water. The sum of the difficulty coefficients of these five parts determines the actual difficulty coefficient of a completed diving action.

(1) Somersault. Somersault (code named $A$ by the International Travel Federation and so on) is the main technical part of the action, so it should be included in the difficulty component. The decisive factor is the number of somersault cycles itself. More cycles are more difficult, and vice versa, followed by height, which will directly affect the completion of the somersault. It can be seen from the FINA rules that the maximum increase in the difficulty coefficient during the transition from $11 / 2$ somersaults to double somersaults is 0.4 , and then the increase in the difficulty coefficient in the $21 / 2$ somersaults is 0.2 , and the increase in the difficulty coefficient for each additional half lap is, respectively, 0.4, 0.2, and 0.8 , which shows that the difficulty coefficient of the tossing situation is not monotonically increasing linearly.

(2) Air Posture. Air posture (B) refers to athletes adopting different postures such as straight, pike, tuck, or any position in the diving process, and the degree of difficulty of the action varies. From the connection point of view, the determining factor of the difficulty of the posture is the posture itself, and the influencing factor is the direction of the jump and the number of tumbling cycles. It is worth noting that the combination of some take-off methods and aerial posture has a negative impact on the degree of difficulty [1].

(3) Rotation. Rotation $(C)$ is also the main technical part of the action. The main determining factor for the difficulty is the number of rotations itself. A larger number of weeks is more difficult, and vice versa. The influencing factors are the take-off method and the tossing situation. The same rotation and in the case of tossing, the difficulty coefficient of arms stand is higher than that of forward, backward, inward, and reflexive take-off [3].
(4) Take-Off Method. The take-off $(D)$ is a forward, reverse dive or a backward, inward, or arm stand dive. The difficulty of the action varies. Therefore, the takeoff method should also be used as the logical composition of the difficulty of the action, and the determining factors are the somersault situation and height. The difficulty coefficient of reflexive tossing is significantly higher than that of forward, backward, and inward tossing. On this basis, the difficulty coefficient of arms stand is further improved. Among them, the difficulty of full gainer of arms stand is significantly higher than that of other somersault situations.

(5) Unnatural Water Entry. The easiest movements to understand, such as turning forward and inward for half somersault, $11 / 2$ somersaults, are considered natural entry in the water, so they do not account for the proportion of the difficulty composition, and vice versa; it is judged as unnatural water entry. If the athlete does not see the surface of the water before entering the water, then it is considered that such entry is logically difficult. The determining factors and difficulty include the direction of the jump and the number of somersault cycles. Moreover, the IAAF stipulates that all movements with twist are uniform. Do not count this item.

\subsubsection{Partial Relevance of Diving Difficulty Coefficient.} The influence and connection of the five parts are visualized here. Each circle in the figure represents the five subparts of the abovementioned difficulty coefficient, and the direction of the arrow indicates the influence among the parts, such as the degree of difficulty of the twist situation $(C)$ being overturned. For the influence of somersault $(A)$ and take-off method $(D)$, the arrows from $A$ and $D$ point to $C$. The drawing is as follows (Figure 1).

If the difficulty coefficient of a complete set of diving actions is recorded as DD, the formula is as follows:

$$
\mathrm{DD}=A+B+C+D+E .
$$

Combining the actual diving action code example and analysis, for example, the difficulty coefficient of the 10meter platform $307 C$ (reverse turning three and a half knees) action is as follows: $3.4=2.7+0+0+0.3+0.4,5257 B$ (backward turning two and a half turns three week and a half). The degree of difficulty of the action is as follows: $4.1=2.1+0.3+1.5+0.2+0$.

\section{The Relationship between the Time for Athletes to Complete Each Diving Action and Their Body Type}

5.1. Problem Analysis. "Once diving and gymnasts start to do a somersault tossing and twist, they often seem to maintain a fixed body type, so rigid bodies can be used to represent these phasors reasonably [7]." The rigidity of the skeleton means that it can be assumed that only by changing the joint angle, the posture change occurs. Therefore, the human body 


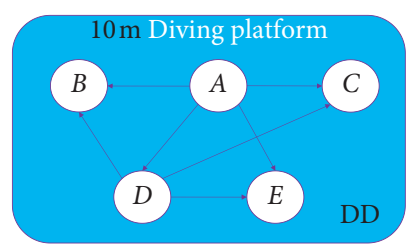

Figure 1: Correlation diagram of components of diving difficulty coefficient.

is easily modeled as a set of rigid segments connected by joints. Therefore, in biomechanics, it is more common to regard the body as a multirigid segment system [8-13].

Therefore, this article firstly derives a version of Euler's equations for deformed rigid bodies from theory [12]. Then, use the two-body model and show how to use this model to achieve a twisting somersault. First, the entire diving process was divided into five stages. Then, the author improved the calculated total time of the five stages achieved in the simulation done by Tong and other researchers. Last, the author used the controlled variable method for the entire diving process, with height and weight being the only two variables. It can be known that height and weight indirectly affect the total time of the entire diving phase by affecting the moment of inertia $[2,14,15]$.

\subsection{Solve the Problem}

5.2.1. Analysis of Athletes Diving in the Air. Air turning and somersaults are the basic movements of springboard divers, and the conservation of angular momentum is the basic physical principle that governs all movements including somersaults and turns [16]. The athlete's head is facing down when doing somersaults as if rotating around an axis from left to right passing through his waist, as shown in Figure 2. In the turning motion, the athlete rotates in the air like a spindle or dancer, similar to rotating around an axis from head to toe. Almost all the most complicated diving moves involve multiple somersaults or turns. After the athlete is in the air, if the resistance of the air is ignored, it will not be affected by the external moment, and its angular momentum should remain unchanged. However, the constant angular momentum means that the product of the angular velocity and the moment of inertia remains unchanged, which does not mean that the angular velocity also remains unchanged.

\subsubsection{Euler Equation Model of Coupled Rigid Body}

(1) Model Establishment. Let the constant angular momentum vector in the spatial fixed frame be 1 . Rigid body dynamics usually use a rigid body frame because the moment of inertia is constant in this frame [17-21]. The change from one coordinate system to another is given by the rotation matrix $R=R(t) \in \mathrm{SO}$ (3), such that $1=\mathrm{RL}$ in the rigid body frame, the vector $L$ is described as a motion vector, and only its length remains unchanged because of $R=R(t) \in \mathrm{SO}(3)$. The angular velocity $\Omega$ in the rigid body frame is a vector of $\Omega \times v=R^{t} \dot{R} v a$ for any vector $v \in R^{3}$.
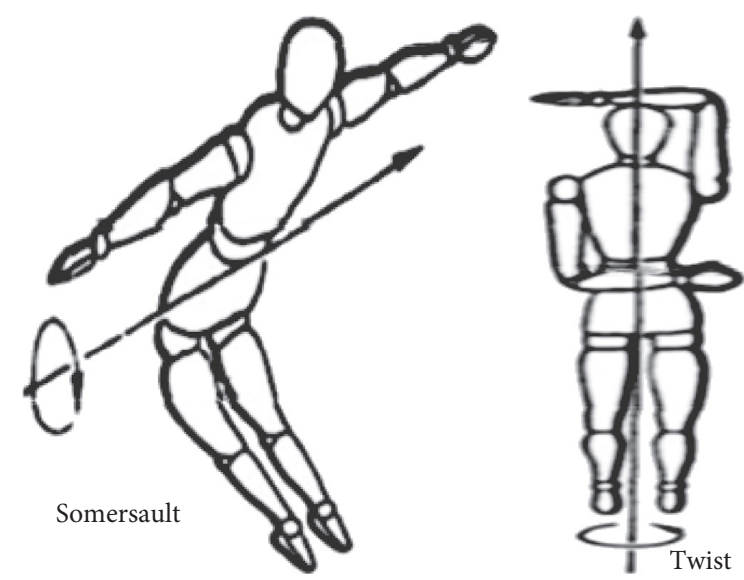

FIgURE 2: Schematic diagram of diving athletes in the air.

Even for a coupled rigid body system, the moment of inertia is usually not constant, and the rigid body frame still gives the simplest motion equation.

The motion equation of the shape-changing body with the angular momentum vector $L \in R^{3}$ in the rigid frame is $\dot{L}=L \times \Omega$, where the angular velocity $L \in R^{3}$ is given by the following formula $\Omega=I^{-1}(L-A)$, where $I=I(t)$ is the moment of inertia and $A=A(t)$ is the "momentum change" produced by the shape change. For the coupled rigid body model, we have

$$
\begin{aligned}
& I=\sum R_{a i} I_{i} R_{a i}^{t}+m_{i}\left(\left|C_{i}\right|^{2} 1-C_{i} C_{i}^{t}\right), \\
& A=\sum\left(m_{i} C_{i} \times \dot{C}_{i}+R_{a i} I_{i} \Omega_{a i}\right),
\end{aligned}
$$

where $m_{i}$ is the mass, $C_{i}$ is the position of the center of mass, $R_{a i}$ is the relative orientation, the relative angular velocity of $\Omega_{a i}$ is such that $\Omega_{a i} \times v=R_{a i}^{t} \dot{R}_{a i} v$ for all $v \in R^{3}$, and the tensor of $I_{i}$ is the inertia of the body $B_{i}$. The sum exceeds all subjects $B_{i}$ including the reference segment.

There are many mathematical models for athletes, and the complexity of each athlete varies. The human body model used can generally be divided into pelvis, abdomen, upper limbs, lower limbs, and other parts according to inertial properties and can be reduced to fewer subdivisions as needed [22]. In this study, it will be assumed that the wrist and ankle joints are fixed. Compared with other parts of the body, the hands and feet are relatively light, so the joint movement can be ignored. Therefore, the athlete can be simplified into a 10-body model, including a torso, a head, two upper arms, two forearms connected to the hands, and two thighs and calves connected to the feet. This is usually the smallest body fragment accepted by a real simulation.

Under the conditions of the research and analysis, in order to make the torsion-tossing model as simple as possible, only a two-body model with a single hinge joint needs to be considered so that the body part except the left arm $B_{I}$ is fixed relative to the body $B_{b}$. In the figure, $C_{b}$ and $C_{I}$ are the centroids of the body and the left arm, respectively, and $C$ is the total centroid. Here, the two-body model shown in Figure 3 is used to study the relationship between the $n$-turn 


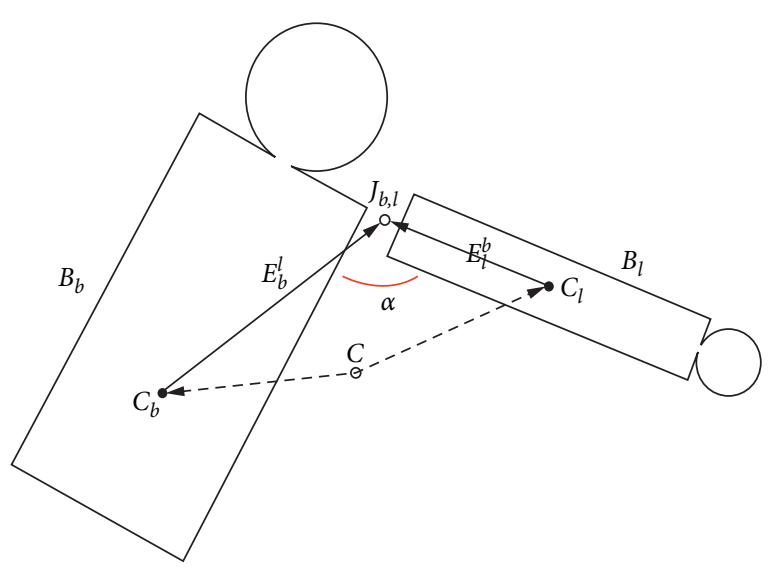

Figure 3: Schematic diagram of two-body.

and $m$-somersault time of divers of different body types (height and weight) and the athlete's height and weight.

In the actual diving process, $m$ is usually a half-integer and $n$ is usually an integer turning forward or inward, or turning backward or reverse. This research will focus on forward turning and tossing, such as $5132 D, 5134 D$, and $5136 D$ (here, only any position is considered for the time being, and bending and tucking will be considered later).

Based on the above analysis, this study will establish a two-body as a theoretical model, in which the change of the moment of inertia I and the change of the angular momentum A are detailed in Bharadwaj et al. [23].

(2) Analysis of the Model. First, we describe the diving program in which the platform diver performs $n$ turns and $m$ somersaults. By using a set of predetermined movements, a simplified two-body model is used for simulation. As shown in Figure 4, the whole set of motion is the left arm abduction, adduction, up, and down movement. The entire simulation process includes 5 stages represented by $S_{n}=(1,2,3,4,5)$, of which the odd stage is a rigid body motion system without deformation (the left arm moves), and the even-numbered stage is a transitional stage with deformation. The specific process is as follows.

The time spent in phase $S_{k}$ is represented by $\tau_{k}$. For convenience, in each individual phase $S_{k}$, we use the relative time $t$ instead of the absolute time from the moment of departure. Moment of inertia, angular momentum, and angular velocity are denoted by $I, L$, and $\Omega$, under the center rigid body frame and denoted by $J, M$, and $W$ under the main shaft frame. Because athletes of different body types have different moments of inertia during diving, the height and weight of athletes are related to the moments of inertia. For the convenience of analysis and comparison, it is assumed that the initial angular momentum of the athletes is the same. Therefore, the angular velocity is assumed to be time $t$ under different frames. Function unless a specific evaluation is performed at a specific time.

The diver uses two key positions for somersault and flip turn. The two key poses are layout and twist. In order to distinguish these two shapes, the time of somersault is expressed as $T^{s}$ and the time of flip turn is expressed as $T^{t}$.
In the stage $S_{1}$, the athlete takes off from the platform at a prescribed vertical speed $V_{0}$ and angular speed $\Omega$. Since the angular velocity is about a principal axis of inertia, it corresponds to a stable rotation with a constant angular momentum $L_{1}$, so the athlete has only somersault. The angular momentum vector remains constant during the take-off and the entire diving process [24].

In the stage $S_{2}$, the athlete prepares for the turning movement. The arm movement causes the rest of the body to rotate in the opposite direction and tilt, which triggers the turning and tossing stage of diving.

In the stage $S_{3}$, the athlete performs flip turn in rigid body motion. In order to make the flip turn analysis easier, we analyze in the main frame. It plays a vital role in the entire diving process because it ensures that the athlete achieves the correct number of turns before returning to somersault.

In the stage $S_{4}$, the athlete performs another transformation at the end of the $S_{3}$ turns and returns to the layout position.

In the stage $S_{5}$, the athlete returns to the pure somersault action and determines the time to ensure that the movement enters the pool in the correct way with the head first into the water. In short, we use $\tau_{3}$ to control the number of turns and use $\tau_{1}+\tau_{5}$ to control the number of somersaults.

The air time of diving includes the sum of the time spent in each of the five stages, namely,

$$
T_{\text {air }}=\tau_{1}+\tau_{2}+\tau_{3}+\tau_{4}+\tau_{5} .
$$

By regulating the vertical speed during take-off, the athlete can control the air time to a certain extent. It is assumed $v_{0}=0$ here for the convenience of calculation.

Using the results of numerical simulation by Bharadwaj et al. [23], when the angular momentum 1 is arbitrary, the result is as follows:

$$
T_{\mathrm{air}}=\tau_{1}+\tau_{2}+\tau_{3}+\tau_{4}+\tau_{5} \approx m T^{s}-0.14339 n T^{t},
$$

where

$$
T^{t}=\frac{2 \pi}{W}=\frac{2 \pi}{l} \frac{J_{y}^{t} J_{z}^{t}}{J_{y}^{t}-J_{z}^{t}} \csc (\rho+x) .
$$

Among them, $T^{s}=2 \pi I^{s} / l$, in order to facilitate our analysis, here $T^{t}=2 \pi I^{t} / l$,

$$
T_{\text {air }}=\frac{2 \pi}{l}\left(m I^{s}-0.14339 n I^{t}\right) .
$$

\subsubsection{Solution and Analysis of the Moment of Inertia Model.} Assuming that the ratio of the athlete's upper and lower body is set to $1: 1$, the length of the bend is $h / 2$ and the length of the tuck is $h / 2[25,26]$.

(1) Moment of Inertia When Bending. When the athlete makes a bend in the air, the schematic diagram is as follows (Figure 5).

Assuming that the athlete's center of mass is located 2/3 away from the foot and rotates around the center of mass, the moment of inertia is 


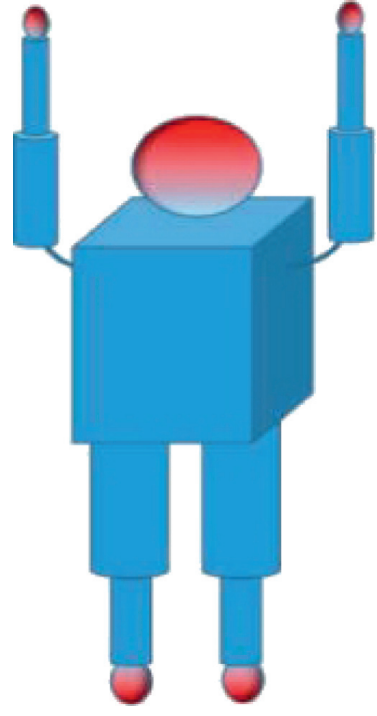

(a)

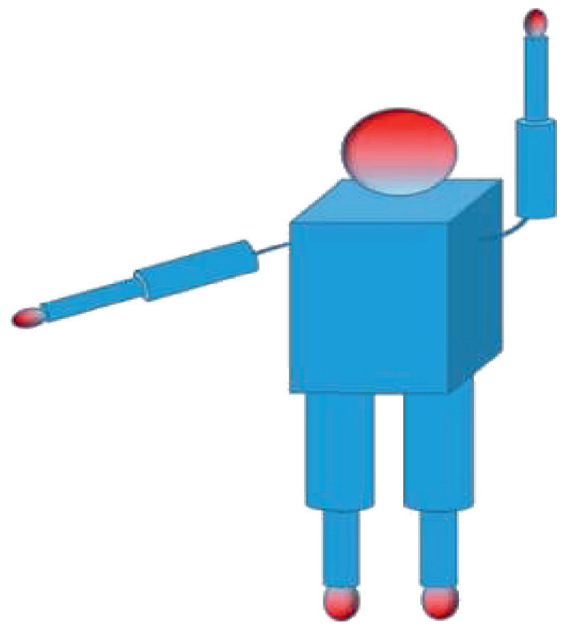

(d)

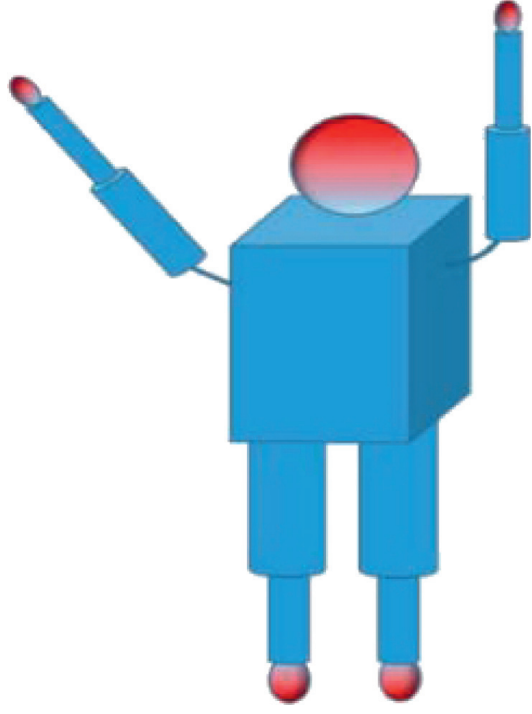

(b)

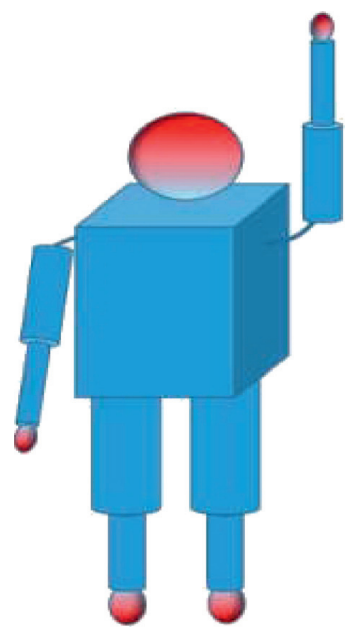

(e)

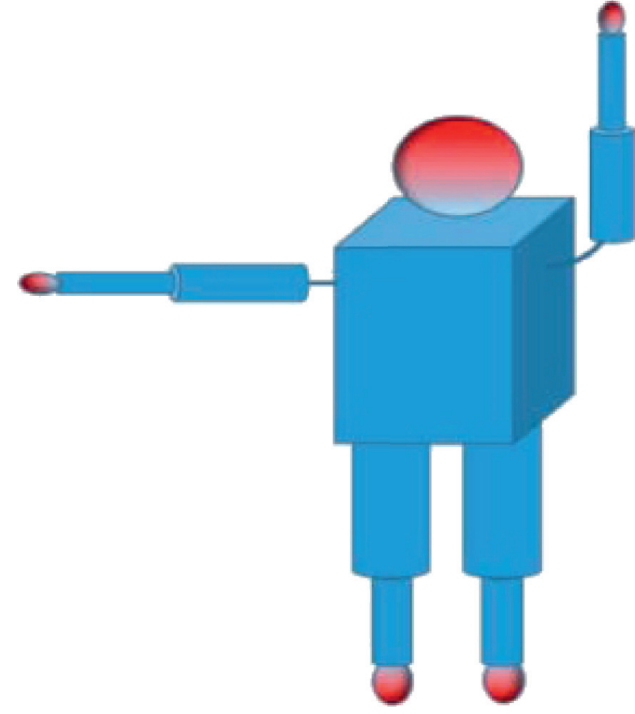

(c)

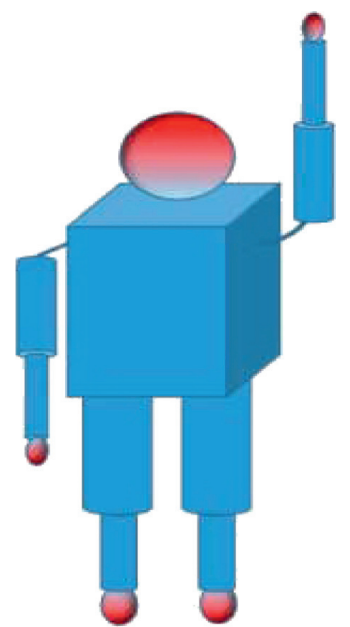

(f)

FIgURE 4: Exploded diagram of diving action: (a) $a=\pi$; (b) $a=2.356$; (c) $a=1.571$; (d) $a=1.047$; (e) $a=0.262$; (f) $a=0$.

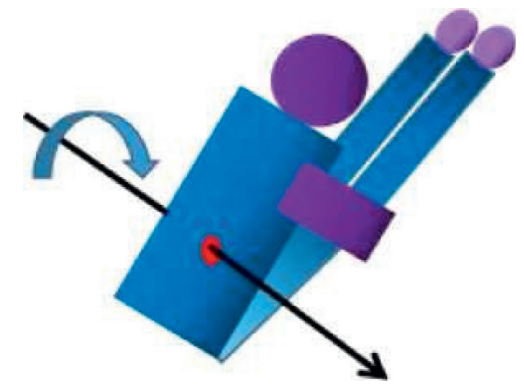

Figure 5: Pike torsion.

$$
I_{1}^{s}=I_{c}+m d^{2}=\frac{1}{9} M l^{2}=\frac{1}{9} M\left(\frac{1}{2} h\right)^{2}=\frac{1}{36} M h^{2} .
$$

(2) Moment of Inertia When Tucked. When the athlete makes a tuck roll in the air, the schematic diagram is as follows (Figure 6).
Assuming that the athlete's center of mass is located $1 / 2$ away from the head and rotates around the center of mass, the moment of inertia is

$$
I_{2}^{s}=\frac{1}{12} M l^{2}=\frac{1}{12} M\left(\frac{1}{2} h\right)^{2}=\frac{1}{48} M h^{2} .
$$

(3) Moment of Inertia during Torsion. When the athlete twists in the air, the schematic diagram is as follows (Figure 7).

The athlete rotates around the length of the body and assumes that the radius is the same as the shoulder length, set at $20 \mathrm{~cm}$, and the moment of inertia is as follows:

$$
I^{t}=\frac{M r^{2}}{2}=\frac{M(0.2)^{2}}{2}=\frac{M}{50} .
$$

According to the formula obtained from the Euler equation model of the coupled rigid body, 


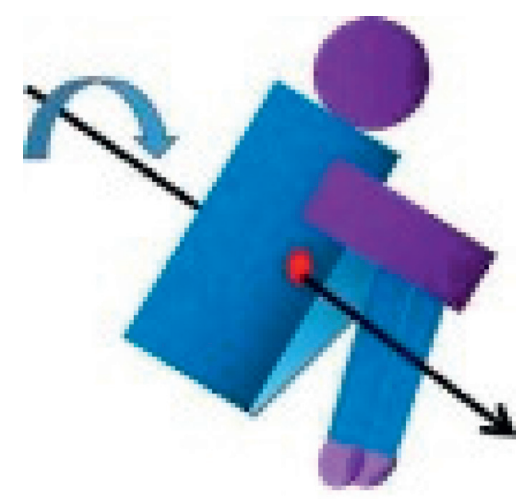

FIgure 6: Tuck.

$$
T_{\mathrm{air}}=\frac{2 \pi}{l}\left(M I^{s}-0.14339 n I^{t}\right)
$$

It can be seen that when the height is higher, the moment of inertia of the tumbling movement and the moment of inertia of the turning movement will increase, but since the coefficient of the former mis much larger than the latter, studies have shown that the turning inertia has "marginal importance," so the turning the influence of inertia is negligible, and overall $T_{\text {air }}$ is increasing [27]. Therefore, the time taken by the athlete to complete each diving action is positively correlated with the athlete's body type.

\section{Construction of the Calculation Model of Body Correction Coefficient}

6.1. Analysis of the Problem. Based on the research on the relationship between the time for the athlete to complete each diving action and the body type, it is concluded that the time for the athlete to complete each diving action is positively correlated with the athlete's body type. Therefore, when the athlete completes the whole set of movements for a relatively long time, the time it takes to adjust the body state to prepare to enter the water is relatively less, and the difficulty of completing the movement is relatively greater, so it is necessary to add body type correction factor.

The entire diving process of the athlete is divided into the air movement part and the body expansion part. Through the analysis of the top three body types of the previous diving World Cup, an optimal body type is found as the standard of the difficulty coefficient of the action to modify the air movement part. Then, it is adjusted by analyzing the vertical posture and splash of the water entering part.

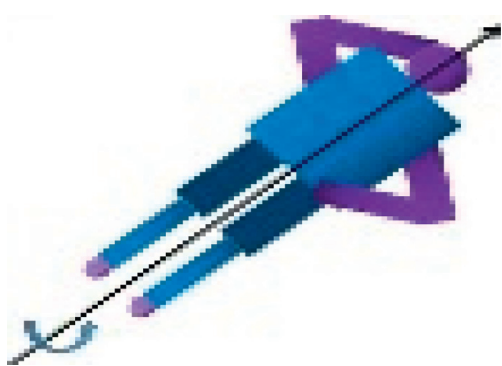

Figure 7: Twist.

6.2. Solve the Problem. First of all, the entire diving process is divided into the air movement part and the body expansion into the water part, and the time spent in the air movement part is recorded as $T_{\text {air }}$. Secondly, corresponding to the calculation formula $\mathrm{DD}=A+B+C+D+E$ of the original difficulty coefficient, the difficulty of the aerial movement part is recorded as $A+B+C$, the difficulty of the take-off part is recorded as $D$, and the difficulty of the body expanding into the water is recorded as $E$. Since it has been assumed that the initial angular momentum of each athlete is the same, the difficulty correction here does not consider the take-off part; that is, the difficulty of the take-off part is $D$ still.

6.2.1. Analysis of the Degree of Difficulty Correction in the Aerial Part of Diving. It can be seen from the competition experience and actual situation that the faster the athlete completes the air movement part of the required air movement, that is, the shorter $T_{\text {air }}$, the time left for the body to prepare for the water movement, and the more conducive to higher completion points.

Therefore, for athletes of different heights and weights, assuming that their initial angular momentum is the same, they satisfy the law of conservation of angular momentum [28-30]:

$$
L=I \Omega \text {. }
$$

Because athletes have different heights and weights, their moments of inertia $I \cdot I$ are different. The greater the height and weight of the athlete, the greater the moment of inertia, and from the law of conservation of angular momentum: the greater the moment of inertia, the lower the athlete's angular velocity, and the longer it takes to complete the required air movement, which is more unfavorable. The water entry action and the influence process are briefly described as follows:

$$
\left\{\begin{aligned}
(h, M) \uparrow & \stackrel{I=}{\longrightarrow} I \uparrow \stackrel{\bar{L}}{\longrightarrow} \Omega \downarrow T_{\text {air }} \uparrow \longrightarrow \text { Difficulty of entering the water } \uparrow, \\
(h, M) \downarrow & \stackrel{\int r^{2} \mathrm{~d} m}{\longrightarrow} I \downarrow \stackrel{\bar{L}}{\longrightarrow} \Omega \uparrow T_{\text {air }} \downarrow \longrightarrow \text { Difficulty of entering the water } \downarrow .
\end{aligned}\right.
$$


From the above analysis, it is known that thin athletes have a body type advantage when doing tossing and turning movements, and body type coefficients should be set for correction. Therefore, this article uses the athlete's air movement time $T_{\text {air }}$ as the correction coefficient to correct the original jumping degree of difficulty.

6.2.2. Modification Analysis of the Difficulty Coefficient of the Diving Body to Expand into the Water Part. From contact with the water surface to the end of all water entry, the size of the splash formed after the athlete enters the water directly affects the final competition result. Therefore, "water pressure" is the key to the water entry stage in diving [31, 32].

Water is a fluid, and it has the basic physical properties of a fluid. According to Archimedes' principle, the water splash formed during diving is due to the fact that the athlete needs to squeeze the same volume of water as the human body out of the water surface when the athlete enters the water, and the water squeezed out of the water surface is splashed out in the form of splashes $[33,34]$.

According to our research, the size of the water splash is mainly related to the following factors:

(1) The Size of the Athlete's Body. Under normal circumstances, the size of the spray is positively related to the size of the athlete's body. Especially for platform jumping athletes, the larger the volume, the more water will be discharged from the water surface when entering the water and the greater the possibility of causing larger splashes. On the contrary, the splash will be smaller.

(2) Water Entry Speed. The faster the water enters, the greater the impact momentum on the water surface. Under the action of the water surface tension, the faster the water spray squeezed out of the water surface will escape, which may make the water splash higher. On the contrary, the water will splash low.

(3) Water-Facing Area. When an athlete enters the water from the air, the larger the contact area between the body and the water surface is, the larger the splash will be. On the contrary, according to the requirements of fluid mechanics, try to make the body enter the water vertically and reduce the water-facing area. It will be smaller.

(4) Body Type. The body type is slender, with wellproportioned muscle lines. The body type with the top and bottom ends is close to streamline, which will form a layer of airflow on the body surface to enhance the viscosity between the body surface and the water flow. Under the action of water surface tension, the displacement is reduced, the water line is elongated, and the splash is reduced. On the contrary, it may produce larger splashes.

From this analysis, we can know that the stage of entering the water is directly related to the athlete's body type.
In addition, the ideal water entry angle should be that the straight body is perpendicular to the horizontal plane. This is related to the air movement time consumed by the water entry adjustment time. This has been considered in the previous correction process. For the convenience of analysis, it is not considered here.

6.2.3. Difficulty Coefficient Correction for the Aerial Part of Diving. Based on the analysis of the body types of the top three in the previous World Diving World Cup, as shown in Table 1, the average height of the top three known men's divers is $1.67 \mathrm{~m}$ and that of women's is $1.55 \mathrm{~m}$. Combined with related research, it is believed that the best diving height is $1.65 \mathrm{~m}$, so this will be used as the standard as the basis for the difficulty of the action [35]. The World Health Organization gives the relationship between body height and weight:

$$
\begin{aligned}
\text { Male: } & (h-80) \times 70 \%=m, \\
\text { Female: } & (h-70) \times 60 \%=m .
\end{aligned}
$$

A male diver with a height of $1.65 \mathrm{~m}$ spends $T_{\text {air }}^{0}$ in the air and uses $T_{\text {air }}^{0}$ when his height increases by $\Delta H$. Without considering the take-off part $D$, the time the athlete spends on the part $A+B+C$ and part $E$ directly affects the difficulty coefficient of the whole process. Therefore, the increase or decrease ratio of $T_{\text {air }}$ to $T_{\text {air }}^{0}$ is used as the correction coefficient. When the difficulty coefficient of the part $A+B+C$ is as follows: $T_{\text {air }}>T_{\text {air }}^{0}$, the difficulty coefficient will increase at this time, namely,

$$
\begin{aligned}
(A+B+C)^{1} & =\left[1+\frac{T_{\text {air }}-T_{\text {air }}^{0}}{T_{\text {air }}^{0}}\right] \cdot(A+B+C)^{0} \\
& =\frac{T_{\text {air }}}{T_{\text {air }}^{0}} \cdot(A+B+C)^{0} .
\end{aligned}
$$

Because the second question gives the conclusion that the time for the athlete to complete each diving action is positively correlated with the athlete's body type, the situation $T_{\text {air }}<T_{\text {air }}^{0}$ is not considered here. In summary, the new difficulty $A+B+C$ after correction is

$$
(A+B+C)^{1}=\frac{T_{\mathrm{air}}}{T_{\mathrm{air}}^{0}} \cdot(A+B+C)^{0} .
$$

6.2.4. Modification of the Coefficient of Difficulty for the Diving Body to Expand into the Water.

$$
E^{1}=\frac{M^{1}}{M^{0}} E^{0}
$$

Therefore, the corrected difficulty coefficient DD of the athlete is finally obtained as

$$
\mathrm{DD}=\frac{T_{\mathrm{air}}}{T_{\mathrm{air}}^{0}} \cdot(A+B+C)^{0}+\frac{M^{1}}{M^{0}} E^{0} .
$$


TABle 1: The heights of the top three in the 14-21 Diving World Cup.

\begin{tabular}{|c|c|c|c|c|c|c|}
\hline \multirow{2}{*}{$\begin{array}{l}\text { Name } \\
(\text { Height/cm) }\end{array}$} & \multicolumn{3}{|c|}{ Male } & \multicolumn{3}{|c|}{ Female } \\
\hline & 1st place & 2nd place & 3rd place & 1st place & 2nd place & 3rd place \\
\hline $14^{\text {th }}$ & $\begin{array}{c}\text { Liang Tian } \\
171 \\
\end{array}$ & $\begin{array}{c}\text { Jia } \mathrm{Hu} \\
170 \\
\end{array}$ & $\begin{array}{c}\text { Despatie } \\
168 \\
\end{array}$ & $\begin{array}{c}\text { Wilkinson } \\
168 \\
\end{array}$ & $\begin{array}{l}\text { Turki } \\
(-)\end{array}$ & $\begin{array}{c}\text { Takiri Miyazaki } \\
(-)\end{array}$ \\
\hline $15^{\text {th }}$ & $\begin{array}{c}\text { Lu-xin Zhou (178) } \\
(-)\end{array}$ & $\begin{array}{c}\text { Yue Lin } \\
157\end{array}$ & $\begin{array}{c}\text { Kravchenko } \\
(-)\end{array}$ & $\begin{array}{c}\text { Tong Jia } \\
150\end{array}$ & $\begin{array}{c}\text { Li-shi Lao } \\
153 \\
\end{array}$ & $\begin{array}{c}\text { Heymans } \\
170 \\
\end{array}$ \\
\hline $16^{\text {th }}$ & $\begin{array}{c}\text { Klein } \\
172 \\
\end{array}$ & $\begin{array}{c}\text { Lu-xin Zhou } \\
178 \\
\end{array}$ & $\begin{array}{c}\text { Boudia } \\
175 \\
\end{array}$ & $\begin{array}{l}\text { Ruo-lin Chen } \\
158\end{array}$ & $\begin{array}{c}\text { Xin Wang } \\
137\end{array}$ & $\begin{array}{c}\text { Paula Esnoza } \\
(-)\end{array}$ \\
\hline $17^{\text {th }}$ & $\begin{array}{c}\text { Mitcham } \\
173\end{array}$ & $\begin{array}{c}\text { Liang Huo } \\
158\end{array}$ & $\begin{array}{c}\text { Bo Qiu } \\
162\end{array}$ & $\begin{array}{c}\text { HU Ya-dan } \\
145\end{array}$ & $\begin{array}{c}\text { CHEN Ruo-lin } \\
158 \\
\end{array}$ & $\begin{array}{c}\text { Li-qun } \mathrm{Wu} \\
152\end{array}$ \\
\hline $18^{\text {th }}$ & $\begin{array}{c}\text { Bo Qiu } \\
162 \\
\end{array}$ & $\begin{array}{c}\text { Milbayev } \\
(-)\end{array}$ & $\begin{array}{c}\text { Walter Fred } \\
(-)\end{array}$ & $\begin{array}{c}\text { Ruo-lin Chen } \\
158 \\
\end{array}$ & $\begin{array}{c}\text { Ya-dan } \mathrm{Hu} \\
145\end{array}$ & $\begin{array}{c}\text { Kortunova } \\
156 \\
\end{array}$ \\
\hline $19^{\text {th }}$ & $\begin{array}{c}\text { Jian Yang } \\
(-)\end{array}$ & $\begin{array}{c}\text { Bo Qiu } \\
162\end{array}$ & $\begin{array}{c}\text { Garsia } \\
(-)\end{array}$ & $\begin{array}{c}\text { Xiao-hui Huang } \\
(-)\end{array}$ & $\begin{array}{c}\text { Hui-xia Liu } \\
(-)\end{array}$ & $\begin{array}{c}\text { Li-qun } \mathrm{Wu} \\
152\end{array}$ \\
\hline $20^{\text {th }}$ & $\begin{array}{c}\text { Bo Qiu } \\
162 \\
\end{array}$ & $\begin{array}{c}\text { Ai-sen Chen } \\
168 \\
\end{array}$ & $\begin{array}{c}\text { Tinsmore } \\
(-)\end{array}$ & $\begin{array}{c}\text { Qian Ren } \\
162 \\
\end{array}$ & $\begin{array}{c}\text { Ya-jie Si } \\
164 \\
\end{array}$ & $\begin{array}{c}\text { Li-qun } \mathrm{Wu} \\
152 \\
\end{array}$ \\
\hline $21^{\mathrm{st}}$ & $\begin{array}{c}\text { Ai-sen Chen } \\
168\end{array}$ & $\begin{array}{c}\text { Jian Yang } \\
(-)\end{array}$ & $\begin{array}{c}\text { Si-mao Ding } \\
(-)\end{array}$ & $\begin{array}{c}\text { Jia-qi Zhang } \\
(-)\end{array}$ & $\begin{array}{c}\text { Qian Ren } \\
162 \\
\end{array}$ & $\begin{array}{c}\text { Pandela } \\
(-)\end{array}$ \\
\hline
\end{tabular}

Note. "(-)" means height data are missing.

\section{Comparative Analysis of the Revised Difficulty Coefficient and the Original Coefficient}

7.1. Research Ideas. First, analyze the constituent elements of each code action of the 10-meter platform, start with the structure of the five difficulty factors of $A, B, C, D, E A, B, C, D, E$, use the revised calculation formula of the athlete's diving difficulty coefficient to modify the difficulty coefficient, and finally add the total difficulty coefficient.

7.2. Research Methods. Import the data in the sample into Excel software; use the revised formula of the athlete's diving difficulty coefficient to calculate the final result.

7.3. Result Analysis. The results are shown in Table 2. The revised difficulty coefficient of each movement has increased significantly, which just confirms the previous conclusion. As the height increases, the weight also increases proportionally. Such athletes perform movements in the air. The more time it takes and the more dominant athletes than the height and weight, the more difficulty they have to overcome. At the same time, the time it takes to prepare to enter the water will be reduced, which will affect the effect of the water pressure and eventually lead to a lower score. And this should be affected by the body type, so the revised difficulty coefficient is fairer than the original difficulty coefficient. Therefore, the original difficulty coefficient is corrected by the body type correction coefficient, and the new difficulty coefficient obtained can more accurately reflect the real difficulty of diving for athletes of different body types.
TABLe 2: Modified table of difficulty coefficient of 10-meter platform jumping.

\begin{tabular}{|c|c|c|c|c|c|c|c|c|c|}
\hline \multirow[b]{2}{*}{$\begin{array}{l}\text { Action } \\
\text { code }\end{array}$} & \multicolumn{2}{|c|}{ Pike } & \multicolumn{2}{|c|}{ Tuck } & \multirow[b]{2}{*}{$\begin{array}{c}\text { Action } \\
\text { code }\end{array}$} & \multicolumn{2}{|c|}{ Pike } & \multicolumn{2}{|c|}{ Tuck } \\
\hline & $\begin{array}{c}\text { Raw } \\
B\end{array}$ & $\begin{array}{c}\text { New } \\
C\end{array}$ & $\begin{array}{c}\text { Raw } \\
B\end{array}$ & $\begin{array}{c}\text { New } \\
C\end{array}$ & & $\begin{array}{c}\text { Raw } \\
B\end{array}$ & $\begin{array}{c}\text { New } \\
B\end{array}$ & $\begin{array}{c}\text { Raw } \\
C\end{array}$ & $\begin{array}{c}\mathrm{New} \\
C\end{array}$ \\
\hline & 23 & 26 & 21 & 24 & & 33 & 37 & 31 & 3.5 \\
\hline & 3 & & & & & & & & 4.1 \\
\hline & & & & & & & & 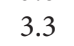 & 3.7 \\
\hline & - & - & 4.7 & & & & & 3.4 & 3.8 \\
\hline & 0 & 3.2 & 2.7 & 3 & & 4.1 & 4.6 & 3.9 & 4.4 \\
\hline & & 8.2 & 33 & & & & & 2.9 & 3.2 \\
\hline 209 & 4.5 & & 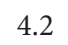 & & & J. & & 3.5 & 3.9 \\
\hline & 3 & 3. & 2.8 & & 5275 & 4.2 & 4.7 & 3.9 & 4.4 \\
\hline & & 4 & 34 & 3. & & 3. & 3. & 3.1 & 3.4 \\
\hline & & & 4. & . & & 3 & & 3.5 & 3.9 \\
\hline & & & 2.5 & 2. & & 3.3 & 3.7 & 3 & 3.3 \\
\hline 4 & 3.5 & 3.9 & 3.2 & 3.6 & 5373 & - & - & 3.6 & 4 \\
\hline 409 & 4.4 & 4.9 & 4.1 & 4.6 & 5375 & - & - & 4 & 4.5 \\
\hline
\end{tabular}

\section{Conclusion}

The results of the study show that the time taken by the athlete to complete each diving action is positively correlated with the athlete's body shape. As the height increases, the weight increases proportionally. The more time such an athlete needs to do in the air, and the less time it takes to prepare to enter the water. Therefore, thin athletes have a body shape advantage. The comparative analysis between the revised difficulty coefficient and the original coefficient shows that the revised difficulty coefficient of each action has increased significantly, and the revised difficulty coefficient takes more into account fairness.

This article combines physical theories and mathematical modeling methods to explain the influence of body type on the time for athletes to complete diving. It provides 
theoretical support for athletes to break through difficult movements in the 10-meter platform and provides athletes and coaches with difficult platform jumping. Scientific training provides the basis.

In real life, the difficulty has the effect of increasing marginal utility, that is, the greater the difficulty of diving, the greater the coefficient of difficulty. This study did not consider the marginal increasing effect of difficulties. Therefore, in the follow-up research, the original difficulty coefficient will be adjusted in grades to realize the optimization of the model.

\section{Data Availability}

The data used to support the findings of this study are included within the article.

\section{Conflicts of Interest}

The authors declare that they have no conflicts of interest.

\section{Acknowledgments}

This research was supported by the Humanities and Social Sciences Research Major Project of Education Department of Anhui Province (SK2019A1161), Anhui Philosophy Social Sciences Planning Project (AHSKY2017D11), and Provincial Foundation for Excellent Young Talents of Colleges and Universities of Anhui Province (gxgwfx2018030).

\section{References}

[1] M. J. Hiley and M. R. Yeadon, "Optimisation of high bar circling technique for consistent performance of a triple piked somersault dismount," Journal of Biomechanics, vol. 41, no. 8, pp. 1730-1735, 2008.

[2] W. Tong and H. R. Dullin, "A new twisting somersault513XD," Journal of Nonlinear Science, vol. 27, no. 6, pp. 2037-2061, 2016.

[3] J. Mikl and D. C. Rye, "Twist within a somersault," Human Movement Science, vol. 45, pp. 23-39, 2016.

[4] M. R. Yeadon, "The biomechanics of the human in flight," The American Journal of Sports Medicine, vol. 25, no. 4, pp. 575-580, 1997.

[5] A. Stewart and L. Sutton, Body Composition in Sport, Exercise, and Health, Taylor \& Francis, London, UK, 2017.

[6] H. R. Wang, H. Ren, and X. L. Xiao, "Preliminary research on morphology characteristic of diving athletein China," China Sport Science and Technology, vol. 44, no. 4, pp. 91-94, 2008.

[7] M. R. Yeadon, "The biomechanics of twisting somersaults Part I: rigid body motions," Journal of Sports Sciences, vol. 11, no. 3, pp. 187-198, 1993.

[8] D. I. Miller, "A computer simulation model of the airborne phase of diving," Ph. D. thesis, Pennsylvania State University, State College, PA, USA, 1970.

[9] N. L. Pike, "Computer simulation of a forward full twisting dive in layout position," Ph. D. thesis, Pennsylvania State University, State College, PA, USA, 1980.

[10] B. Van Gheluwe, "A biomechanical simulation model for airbourne twist in backward somersaults," Journal of Human Movement Studies, vol. 7, pp. 1-22, 1981.
[11] H. Hatze, "A mathematical model for the computational determination of parameter values of anthropomorphic segments," Journal of Biomechanics, vol. 13, no. 10, pp. 833-843, 1980.

[12] R. L. Huston, Principles of Biomechanics, CRC Press, Taylor \& Francis Group, Boca Raton, FL, USA, 2009.

[13] M. R. Yeadon and M. J. Hiley, "The control of twisting somersaults," Journal of Biomechanics, vol. 47, no. 6, pp. 1340-1347, 2014.

[14] H. R. Dullin and W. Tong, "Twisting somersault," SIAM Journal on Applied Dynamical Systems, vol. 15, no. 5, pp. 1806-1822, 2016.

[15] W. Tong, "Coupled rigid body dynamics with application to diving," Ph. D. thesis, University of Sydney, Sydney, Australia, 2015.

[16] M. R. Yeadon and M. J. Hiley, "Twist limits for late twisting double somersaults on trampoline," Journal of Biomechanics, vol. 58, no. 5, pp. 174-178, 2017.

[17] J. F. Zhang, J. J. Liu, O. F. Gong, W. J. Yuan, and Y. X. Zhang, "Calculation on rotary inertia for homogeneous cylindrical shell rigid body," Journal of Jilin Normal University, vol. 36, no. 2, pp. 84-86, 2015.

[18] K. C. Yang, "The formula for calculating the moment of inertia of a homogeneous cylindrical rigid body with respect to any axis," Mechanics in Engineering, vol. 5, pp. 53-54, 1983.

[19] R. X. Zhou, "Research on the rotational inertia of several categories of rigid-body," Journal of Guiyang University (Natural Sciences), vol. 6, no. 3, pp. 10-17, 2011.

[20] Y. J. Qiu and J. S. Li, "Calculation of moment of inertia based on height and weight," Zhejiang Sport Science, vol. 6, pp. 38-40, 1994.

[21] R. Montgomery, "How much does the rigid body rotate? A Berry's phase from the 18th century," American Journal of Physics, vol. 59, no. 5, pp. 394-398, 1998.

[22] M. R. Yeadon, "Mechanics of twisting somersaults," $\mathrm{Ph}$. D. thesis, Loughborough University of Technology, Loughborough, UK, 1984.

[23] S. Bharadwaj, N. Duignan, H. R. Dullin, K. Leung, and W. Tong, "The diver with a rotor," Indagationes Mathematicae, vol. 27, no. 5, pp. 1147-1161, 2016.

[24] C. Frohlich, "Do springboard divers violate angular momentum conservation?" American Journal of Physics, vol. 47, no. 7, pp. 583-592, 1979.

[25] M. R. Yeadon, "The biomechanics of twisting somersaults Part III: aerial twist," Journal of Sports Sciences, vol. 11, no. 3, pp. 209-218, 1993.

[26] M. R. Yeadon, "The biomechanics of twisting somersaults Part IV: partitioning performances using the tilt angle," Journal of Sports Sciences, vol. 11, no. 3, pp. 219-225, 1993.

[27] J. Mikl, "Limits of aerial techniques when performing somersaults," Ph. D. thesis, The University of Sydney, Sydney, Australia, 2015.

[28] C. Y. Wang and S. P. Gan, "Application of the law of conservation of angular momentum," College Physics, vol. 5, pp. 12-13, 1988.

[29] O. X. Gao, M. Wan, Z. K. Pan, and Y. C. Yang, "Application of conservation law of angular momentum in plane motion of rigid body," Journal of Zunyi Normal University, vol. 11, no. 5, pp. 60-62, 2009.

[30] D. L. Fan, “Adult standard scale algorithm," Chinese Journal of Nursing, vol. 9, p. 409, 1992.

[31] H. Q. Jin, H. Wei, and S. N. Zhang, "Experimental research on splash-eliminating movement of diving," Journal of Shanghai University of Sport, vol. 29, no. 4, pp. 49-52, 2005. 
[32] L. Chen, "Analysis on the technique of water spray in water inlet of diver," Swimming, vol. 4, pp. 62-63, 2014.

[33] G. Juarez, T. Gastopoulos, Y. Zhang, M. L. Siegel, and P. E. Arratia, "Splash control of drop impacts with geometric targets," Physical Review. E, Statistical, Nonlinear, and Soft Matter Physics, vol. 85, no. 2, Article ID 026319, 2012.

[34] J.-G. Qian, S. Zhang, and H.-q. Jin, "ComputerSimulation of slash control'in competitive diving," in Proceedings of Swim Sessions: XIX International Symposium on Biomechanics in Sports, pp. 113-116, San Francisco, CA, USA, June 2001.

[35] Y. Zhang, R. H. Wang, and Y. Chen, "On the current situation and development of the research on diving talent selection in China," Journal of Beijing Sport University, vol. 31, no. 12, pp. 1666-1670, 2008. 